\title{
OCLUSÃO VASCULAR E SUA APLICAÇÃ̃o NA PRÁTICA CLÍNICA
}

Antes de sua aplicação na prática clínica, alguns fatores devem ser considerados. Uma escala de percepção de pressão de 0 a 10 pode ser utilizada para quantificar o nível de pressão do manguito durante o treinamento com oclusão vascular para os membros inferiores e superiores. Uma percepção de 0 significa nenhuma pressão, uma percepção de pressão de 6-7 demonstra oclusão das veias, mas não das artérias e uma percepção de 10 é descrita como dor severa e oclusão das veias e artérias. Um exemplo da aplicabilidade prática da escala é apresentado na Figura 1, adaptada de estudos anteriores (WILSON et al., 2013; LOWER et al., 2014). É importante lembrar que uma percepção de 6-7 corresponde a $80 \%$ de oclusão das veias, mas não das artérias.

Figura 1 Escala de percepção de pressão para o método oclusão vascular aplicado durante o treinamento resistido

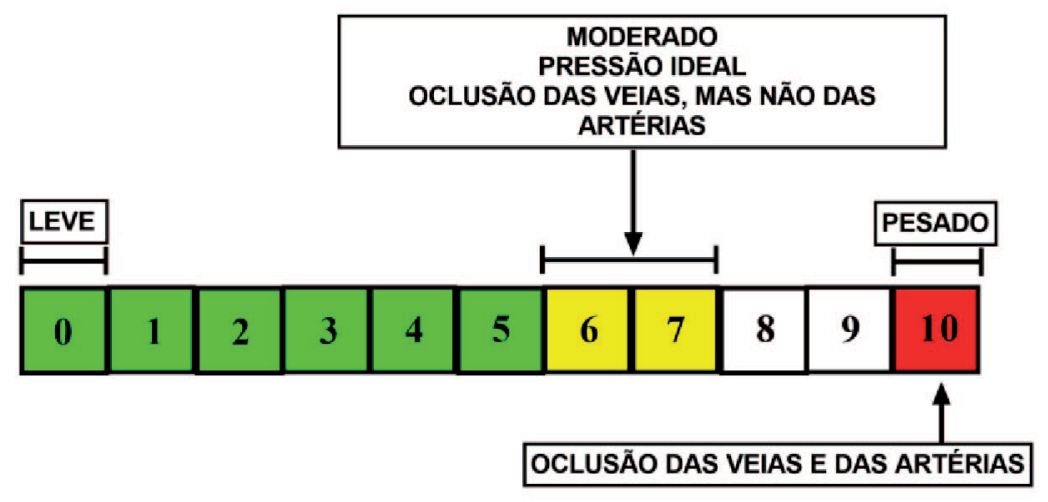


Antes de aplicar o método oclusão vascular, é importante medir as circunferências dos braços e das pernas. A circunferência do braço será medida no nível acromial-radial médio e a da coxa será medida $1 \mathrm{~cm}$ abaixo do nível da dobra glútea para a determinação correta do tamanho do manguito que será utilizado (NORTON; OLDS; ALBERNAZ, 2005). O posicionamento dos manguitos para membros superiores é na parte proximal do braço perto da axila e nos membros inferiores é na parte proximal da coxa perto da dobra inguinal (Figura 2).

Figura 2 Posicionamento dos manguitos no membro superior e inferior

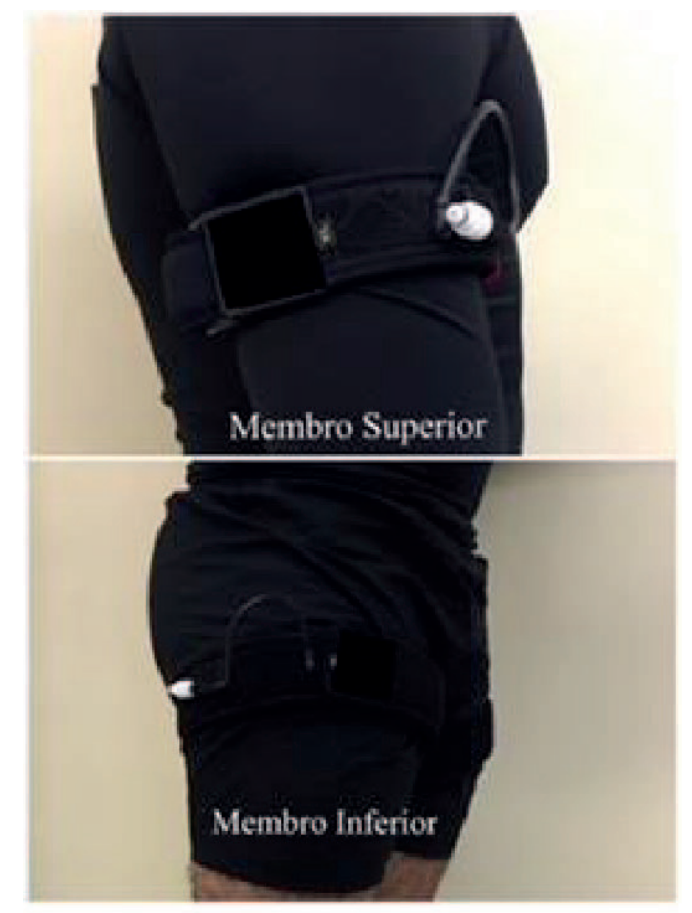

Considerando que o treinamento envolve a aplicação de um material específico, tipo um manguito pneumático próximo ao músculo onde o estímulo será aplicado. Infelizmente, devido ao custo do equipamento para grande parte da população o acesso será dificultado. Um dos equipamentos ou manguitos criados mais indicados para a aplicação do método oclusão vascular para braços e pernas é da marca Kaatsu, Sato Sports Plaza, Tóquio, Japão. No entanto, manguitos adaptados e vendidos no Brasil podem ser utilizados desde que se considere as circunferências do braços e pernas (Tabela 1), avaliações dos fatores de risco e utilização da tabela de percepção de pressão antes da aplicação do método. 
Após o posicionamento adequado dos manguitos e sobre a roupa e não diretamente sobre a pele, familiarize o indivíduo com a pressão exercida pelo manguito. Com isso, infle o manguito até $120 \mathrm{mmHg}$ e mantenha por 30 segundos. Libere a pressão por 10 segundos e depois infle para uma pressão de $140 \mathrm{mmHg}$ e mantenha por 30 segundos. Libere novamente a pressão por 10s. Faça isso até que se alcance a pressão de $160 \mathrm{mmHg}$ com aumentos de $20 \mathrm{mmHg}$ entre cada inflação e desinflação (ABE et al., 2009; FUKUDA et al., 2013; RENZI; TANAKA; SUGAWARA, 2010). Aumentos de $10 \mathrm{mmHg}$ podem ser realizados a cada dia até o oitavo dia (ABE; KEARNS; SATO, 2006). Uma vez que os manguitos estejam inflados, eles continuam até o final da sessão de treinamento com duração máxima de 15 minutos para membros superiores e 20 minutos para membros inferiores.

É muito importante que durante a ótima pressão onde teremos oclusão das veias, mas não das artérias. $\mathrm{O}$ aluno deve sempre apresentar perfusão capilar e isso pode ser verificado ao pressionar a ponta ou porção distal do dedo ou unha e observar se o retorno sanguíneo é inferior a 3 segundos. A coloração de sua pele em seus membros deve ser sempre rosa ou vermelho robusto. Além disso, o cliente deve sentir pulsações embaixo do manguito pneumático. Quando houver perfusão capilar superior a 6 segundos e pele pálida, isso pode ser um indicativo que houve oclusão total das veias e artérias e o treinamento deverá ser interrompido imediatamente.

Tabela 1 Tamanhos apropriados das bandas ou manguitos para serem utilizados

\begin{tabular}{|ll|}
\hline Circunferência dos braços & Circunferência das pernas \\
\hline - Pequeno: $18-28 \mathrm{~cm}$ & - Pequeno: $40-50 \mathrm{~cm}$ \\
- Moderado: $28-38 \mathrm{~cm}$ & - Moderado: $50-60 \mathrm{~cm}$ \\
- Grande: $38-48 \mathrm{~cm}$ & - Grande: $60-70 \mathrm{~cm}$ \\
\hline
\end{tabular}

Abaixo seguem as Tabelas 2 e 3, adaptada de estudos anteriores (LOENNEKE et al., 2012; LOENNEKE et al., 2013; SCOTT et al., 2015; SLYSZ; STULTZ; BURR, 2016) sobre recomendações para a aplicação do método oclusão vascular durante o treinamento resistido e caminhada para aumentos da força muscular e hipertrofia durante a prática clínica. 
Tabela 2 Recomendações para aplicação do método oclusão vascular durante o treinamento resistido.

\begin{tabular}{|c|c|}
\hline Variáveis & Recomendações \\
\hline Aplicação do manguito & Próximo ao membro onde será aplicado. \\
\hline Tipo de manguito & $\begin{array}{l}\text { Manguitos largos para as coxas }(6-13,5 \mathrm{~cm}) \text { e manguitos } \\
\text { estreitos para os braços }(3-6 \mathrm{~cm}) \text {. } \\
\text { Manguitos infláveis: } 50-80 \% \text { de pressão para oclusão do } \\
\text { fluxo sanguíneo da artéria em repouso. }\end{array}$ \\
\hline Tipo de exercício & Exercício uniarticulares e multiarticulares. \\
\hline Carga no exercício & Exercícios com baixa carga (20-40\% 1RM). \\
\hline Volume de treinamento & $\begin{array}{l}50-80 \text { repetições por exercício (séries não precisam ser } \\
\text { realizadas até falha). }\end{array}$ \\
\hline $\begin{array}{l}\text { Intervalo de recuperação entre } \\
\text { os exercícios. }\end{array}$ & $30-45$ segundos. \\
\hline Frequência de treinamento. & $\begin{array}{l}\text { Populações clínicas: } 2-3 \text { sessões por semana. } \\
\text { Atletas: } 2-4 \text { sessões por semana. } \\
\text { Ganhos de hipertrofia são superiores quando o treinamento } \\
\text { é realizado } 3 \text { vezes na semana quando comparado com duas. }\end{array}$ \\
\hline Tempo de treinamento & $\begin{array}{l}\text { Quando o treinamento aeróbio for combinado com oclusão } \\
\text { vascular, treinos com tempo de duração > que } 6 \text { semanas } \\
\text { são superiores para ganhos de força muscular. } \\
\text { Ganhos de hipertrofia são superiores quando o treinamento } \\
\text { tem duração > que } 8 \text { semanas de treinamento. }\end{array}$ \\
\hline Pressão & $\begin{array}{l}\text { Ganhos de força muscular foram superiores quando a } \\
\text { pressão no manguito foi > que } 150 \mathrm{mmHg} \text {. }\end{array}$ \\
\hline $\begin{array}{l}\text { Pressão no manguito de acordo } \\
\text { com a circunferência da coxa }\end{array}$ & $\begin{array}{l}\text { < } 45-50 \mathrm{~cm}: 120 \mathrm{mmHg} \text {. } \\
51-55 \mathrm{~cm}: 150 \mathrm{mmHg} \text {. } \\
56-59 \mathrm{~cm}: 180 \mathrm{mmHg} \text {. } \\
\geq 60 \mathrm{~cm}: 2010 \mathrm{mmHg} \text {. } \\
\text { A circunferência da coxa é um fator determinante para a } \\
\text { pressão de oclusão sobre a artéria. Além disso, o uso da } \\
\text { pressão arterial sistólica para determinação do nível de } \\
\text { pressão de oclusão atualmente é questionado. }\end{array}$ \\
\hline $\begin{array}{l}\text { Duração da sessão de treinamento } \\
\text { com o método oclusão vascular }\end{array}$ & $\begin{array}{l}\text { Não exceder } 15 \text { minutos para membros superiores e } 20 \\
\text { minutos para membros inferiores. }\end{array}$ \\
\hline
\end{tabular}


Tabela 3. Pontos importantes para aplicação do método oclusão vascular durante o treinamento resistido.

\begin{tabular}{|c|c|}
\hline Variáveis & Pontos importantes \\
\hline Aplicação do manguito & Não aplicar diretamente sobre a pele. \\
\hline Tipo de manguito & $\begin{array}{l}\text { Circunferência do membro: grandes membros requerem } \\
\text { alta pressão. } \\
\text { Largura do manguito: manguitos largos alcançam oclusão } \\
\text { com baixas pressões quando comparados com manguitos } \\
\text { estreitos }\end{array}$ \\
\hline Tipo de exercício & $\begin{array}{l}\text { O tipo de exercício que pode ser tolerado pelo aluno deve } \\
\text { ser considerado antes de aplicar o método. }\end{array}$ \\
\hline Carga no exercício & $\begin{array}{l}\text { Séries múltiplas de baixa carga e com oclusão vascular } \\
\text { promovem estímulos metabólicos similares ao treinamento } \\
\text { resistido de alta intensidade. }\end{array}$ \\
\hline Volume de treinamento & Padrão esquemático de 30-15-15-15 repetições. \\
\hline $\begin{array}{l}\text { Intervalo de recuperação entre } \\
\text { os exercícios. }\end{array}$ & $\begin{array}{l}\text { A oclusão deve ser mantida durante o intervalo de } \\
\text { recuperação. }\end{array}$ \\
\hline Frequência de treinamento. & Pode ser treinado duas vezes por dia. \\
\hline Tempo de treinamento & $\begin{array}{l}\text { Para a velocidade da caminhada, ganhos de força são } \\
\text { superiores quando a intensidade é }>\text { que } 70 \text { metros por } \\
\text { minuto. }\end{array}$ \\
\hline Pressão & $\begin{array}{l}\text { Uso de pressão personalizada baseada na pressão de oclusão } \\
\text { do membro do aluno. Ademais, uso da restrição moderado } \\
\text { do fluxo sanguíneo entre } 40 \text { a } 80 \% \text { da pressão de oclusão } \\
\text { do membro. }\end{array}$ \\
\hline $\begin{array}{l}\text { Pressão no manguito de acordo } \\
\text { com a circunferência da coxa }\end{array}$ & $\begin{array}{l}\text { Nesse estudo o percentual da carga foi de } 30 \% \text { de } 1 \mathrm{RM} \text { e } \\
\text { foi usado } 60 \% \text { de oclusão da artéria. } \\
\text { Estudos ainda são necessácios para examinar a eficácia } \\
\text { dessas recomendações. Além disso, guias específicos para } \\
\text { membros superiores ainda não foram publicados. }\end{array}$ \\
\hline $\begin{array}{l}\text { Duração da sessão de } \\
\text { treinamento com o método } \\
\text { oclusão vascular }\end{array}$ & $\begin{array}{l}\text { Os usuários podem sentir um leve formigamento nos dedos } \\
\text { das mãos e dos pés durante o treinamento com oclusão } \\
\text { vascular. No entanto, o treino deve ser interrompido } \\
\text { imediatamento se o aluno sentir tontura ou dormência dos } \\
\text { membros. }\end{array}$ \\
\hline
\end{tabular}


Para a prescrição do treinamento resistido com oclusão vascular, podemos verificar que até o momento utiliza-se o percentual de 1RM. O teste de 1RM (a maior quantidade de carga levantada durante uma única repetição) tem sido reconhecida como um procedimento padrão e apesar de ser aceito em populações de todas as idades, poucos estudos tem reportado informações importantes sobre sua segurança em idosos. Com isso, precauções adicionais são essenciais nessa população ou indivíduos com inabilidade de levantar cargas elevadas.

Considerando que o teste de 1RM pode induzir instabilidades hemodinâmicas ou lesões musculoesqueléticas em idosos (DE VOS et al., 2008; POLLOCK et al., 1991; SHAW et al., 1995). As adaptações promovidas pelos estudos de Shimizu et al. (2016) e Cook et al. (2017) estimando a carga de 1RM usando o número de repetições submáximas ( $<10$ repetições) pode ser considerada uma ótima alternativa através de equações (BRZYCKI, 1993) para a prescrição de treinos baseados no percentual de $1 \mathrm{RM}$.

Por exemplo, se 8 repetições foram realizadas com uma carga de $56 \mathrm{~kg}$, o cálculo a seguir poderia ser utilizado para estimar $1 \mathrm{RM}$ na cadeira extensora e depois $30 \%$ de 1 RM.

Estimativa de 1RM: carga submáxima $(\mathrm{kg}) / 102,78$ - 2,78 x número de repetiçōes) /100 (BRZYCKI, 1993).

$$
\begin{gathered}
1 \mathrm{RM}=102,78-2,78 \text { (repetiçōes) } \\
1 \mathrm{RM}=102,2,78(8 \text { repetiçōes }) \\
1 \mathrm{RM}=102,78-22,24 \\
1 \mathrm{RM}=80,54 / 100 \\
1 \mathrm{RM}=56 \mathrm{~kg} / 0,8054 \\
1 \mathrm{RM}=69,53 \mathrm{~kg} .
\end{gathered}
$$

É importante notar que a equação nos estudos supracitados foi utilizada para a prescrição do treinamento com oclusão vascular utilizando os exercícios cadeira extensora, leg press, supino e remada (COOK et al., 2017; SHIMIZU et al., 2016).

É muito importante que o profissional da área da saúde considere também para qual tipo de exercício e população que a equação de estimação de 1RM foi validada, não significando que esta possa ser utilizada em qualquer indivíduo ou exercício físico. 


\section{REFERÊNCIAS BIBLIOGRÁFICAS}

ABE, T. et al. Skeletal muscle size and strength are increased following walk training with restricted leg muscle blood flow: implications for training duration and frequency. International Journal of KAATSU Training Research, v. 5, n. 1, p. 9-15, 2009.

ABE, Takashi; KEARNS, Charles F.; SATO, Yoshiaki. Muscle size and strength are increased following walk training with restricted venous blood flow from the leg muscle, Kaatsu-walk training. Journal of applied physiology, v. 100, n. 5, p. 1460-1466, 2006.

BRZYCKI, Matt. Strength testing-predicting a one-rep max from reps-to-fatigue. Journal of Physical Education, Recreation \& Dance, v. 64, n. 1, p. 88-90, 1993.

COOK, Summer B. et al. Blood flow restricted resistance training in older adults at risk of mobility limitations. Experimental gerontology, v. 99, p. 138-145, 2017.

DE VOS, Nathan J. et al. Continuous hemodynamic response to maximal dynamic strength testing in older adults. Archives of physical medicine and rehabilitation, v. 89, n. 2, p. 343-350, 2008.

FUKUDA, T. et al. Low-intensity kaatsu resistance exercises using an elastic band enhance muscle activation in patients with cardiovascular diseases. International Journal of KAATSU Training Research, v. 9, n. 1, p. 1-5, 2013.

LOENNEKE, Jeremy P. et al. Blood flow restriction does not result in prolonged decrements in torque. European journal of applied physiology, v. 113, n. 4, p. 923-931, 2013.

LOENNEKE, Jeremy P. et al. Effects of cuff width on arterial occlusion: implications for blood flow restricted exercise. European journal of applied physiology, v. 112, n. 8, p. 2903-2912, 2012.

LOWERY, Ryan P. et al. Practical blood flow restriction training increases muscle hypertrophy during a periodized resistance training programme. Clinical physiology and functional imaging, v. 34, n. 4, p. 317-321, 2014.

NORTON, Kevin; OLDS, Tim; ALBERNAZ, Nilda Maria Farias de. Antropométrica: um livro sobre medidas corporais para o esporte e cursos da área de saúde. In:

Antropométrica: um livro sobre medidas corporais para o esporte e cursos da área de saúde. 2005.

POLLOCK, Michael L. et al. Injuries and adherence to walk/jog and resistance training programs in the elderly. Medicine and Science in Sports and Exercise, v. 23, n. 10, p. 1194-1200, 1991.

RENZI, Christopher P.; TANAKA, Hirofumi; SUGAWARA, Jun. Effects of leg blood flow restriction during walking on cardiovascular function. Medicine and science in sports and exercise, v. 42, n. 4, p. 726, 2010.

SCOTT, Brendan R. et al. Exercise with blood flow restriction: an updated evidencebased approach for enhanced muscular development. Sports medicine, v. 45, n. 3, p. 313-325, 2015. 
SHAW, Carl E.; MCCULLY, Kevin K.; POSNER, Joel D. Injuries during the one repetition maximum assessment in the elderly. Journal of cardiopulmonary rehabilitation, v. 15, n. 4, p. 283-287, 1995.

SHIMIZU, Ryosuke et al. Low-intensity resistance training with blood flow restriction improves vascular endothelial function and peripheral blood circulation in healthy elderly people. European journal of applied physiology, v. 116, n. 4, p. 749-757, 2016.

SLYSZ, Joshua; STULTZ, Jack; BURR, Jamie F. The efficacy of blood flow restricted exercise: A systematic review \& meta-analysis. Journal of science and medicine in sport, v. 19, n. 8, p. 669-675, 2016.

WILSON, Jacob M. et al. Practical blood flow restriction training increases acute determinants of hypertrophy without increasing indices of muscle damage. The Journal of Strength \& Conditioning Research, v. 27, n. 11, p. 3068-3075, 2013. 